\title{
A Remark on the Aharonov-Bohm Potential and a Discussion on the Electric Charge Quantization
}

\author{
F. A. Barone* \\ $C B P F$, Rio de Janeiro, Brazil \\ E-mail: fbaronedcbpf.br \\ J. A. Helayël-Neto \\ CBPF, Rio de Janeiro, Brazil \\ E-mail: helayelecbpf.br
}

\begin{abstract}
The purpose of this work is to stress on a mathematical requirement of the Stokes' theorem that, naturally, yields a reassessment of the electric charge quantization condition, which is, here, explicitly carried out in the context of the Aharonov-Bohm set-up. We argue that, by virtue of an ambiguity in the definition of the circulation of the vector potential, a modified quantization condition comes out for the electric charge that opens the way for understanding fundamental fractional charges. One does not, any longer, need to rely on the existence of a magnetic monopole to justify electric charge quantization.
\end{abstract}

Fourth International Winter Conference on Mathematical Methods in Physics 09 - 13 August 2004

Centro Brasileiro de Pesquisas Fisicas (CBPF/MCT), Rio de Janeiro, Brazil

${ }^{*}$ Speaker. 


\section{Introduction}

After Dirac's seminal papers on magnetic monopoles [1], there appeared several others in the literature [2, 3], all of them presenting quantization conditions for the electric charge, but involving magnetic monopoles as well. We aim here at reassessing charge quantization in the absence of monopoles. To do that, we reconsider the Aharonov-Bohm experiment on diffraction of charged particles.

In their celebrated papers of 1959 and 1961, Aharonov and Bohm [4, 5] considered an infinitely long solenoid inside which we could have a uniform and constant magnetic field; outside no magnetic field is present. Outside the solenoid, Aharonov and Bohm took a vector potential in such a way that its circulation around a closed path would give the magnetic flux through a surface whose the boundary was the considered path.

In this work, we show that it is not conflicting with Stokes' Theorem to take other choices to the vector potential outside the solenoid, even if their circulations around a closed path do not give the flux of the magnetic field through a surface whose boundary is the considered path. If we adopt this possibility (that is valid for the Classical Mechanics), in the context of Quantum Mechanics, and slightly and conveniently modify the potential chosen by Aharonov and Bohm, we are straight taken to a quantization condition for the possible values of the electric charges in the nature, with no need to appeal for the existence of magnetic monopoles.

The modification we consider in the Aharonov-Bohm choice for the vector potential can be interpreted as if we had taken an Aharonov-Bohm-like potential as being a pure gauge field. The quantization condition obtained is consistent with the quark fractional charges and with the existence of anti-particles.

\section{Quantization condition}

The basic set-up of the Aharonov-Bohm effect is an infinitely long solenoid of radius $R$ where, in its interior, we have a uniform and constant magnetic field; in its exterior, a null magnetic field. One considers that no particles, or wave functions, can penetrate in the interior of the solenoid.

In cylindrical coordinates, the magnetic field in the whole space can be written as

$$
\mathbf{B}(\rho, \phi, z)= \begin{cases}\mathbf{B}_{I}=B \hat{z}, & \rho<R \\ \mathbf{B}_{E}=\mathbf{0}, & \rho>R\end{cases}
$$

where $\phi$ is the azimuthal angle, and we considered the solenoid axis lying on the $\mathbf{z}$ axis. On the solenoid, $\rho=R$, the field is not defined.

In the interior of the solenoid, we can have a vector potential given by $\mathbf{A}_{I}=B \rho / 2 \hat{\phi}$. In the exterior of the solenoid, we can choose any vector potential, $\mathbf{A}_{E}$, whose rotational is zero. In the context of Classical Electrodynamics, an interesting choice is a vector potential of the form

$$
\mathbf{A}(\rho, z, \phi)=\left\{\begin{array}{l}
\mathbf{A}_{I}(\rho, z, \phi)=\frac{B \rho}{2} \hat{\phi}, \rho<R \\
\mathbf{A}_{E}(\rho, z, \phi)=\frac{\gamma}{\rho} \hat{\phi}, \rho>R
\end{array} .\right.
$$




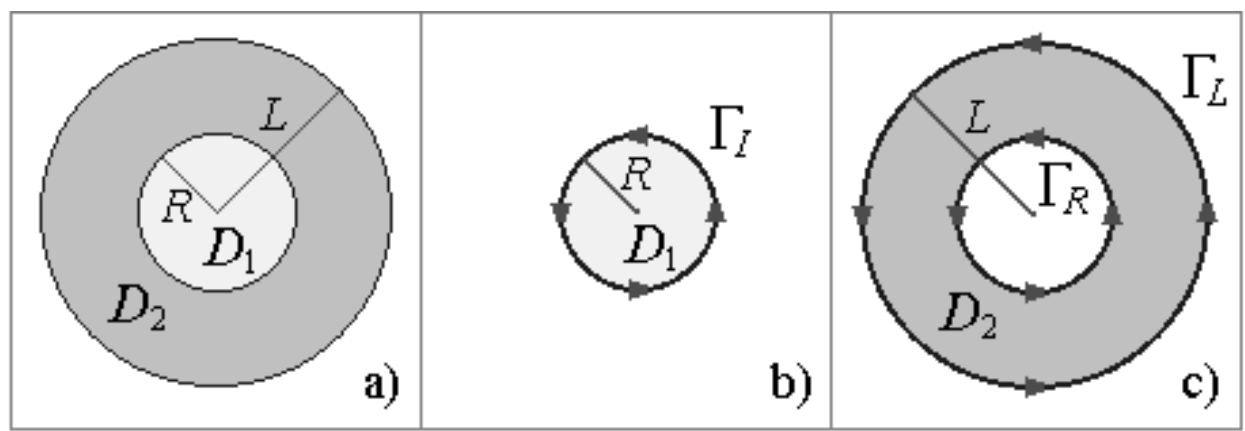

Figure 1: a) Region $D$ divided into two others, $D_{1}$ and $D_{2}$, where the vector potential is of class $C^{1}$. b) Region $D_{1}$ with the path $\Gamma_{I}$ in its boundary. c) region $D_{2}$ with the paths $\Gamma_{L}$ and $\Gamma_{R}$ in its external and internal boundaries, respectively.

where $\gamma$ is a constant. In the exterior region, the potential (2.2) has the property that its integral along any circular path perpendicular to the $z$-axis, and centered at the origin, does not depend on the path radius:

$$
\oint_{\Gamma} \mathbf{A}_{E} \cdot d \ell=2 \pi \gamma
$$

for any circular path $\Gamma$ in the exterior region (for $r>R$ ), where $d \ell$ stands for the tangent vector to the curve.

In order to calculate the flux of the magnetic field (2.1) through a disc $D$ of radius $L>R$ lying on the $x y$ plane and centered at the origin, we can use Stokes' Theorem, but taking into account two points: (i) the vector potential (2.2) is not of class $C^{1}$ [6], due to its behavior at $\rho=R$, and (ii) we are using cylindrical coordinates, which is not a one-to-one map on the region we want to calculate the flux. To avoid the problem presented in (i), we divide the disc $D$ into two regions: the first one, $D_{1}$, is the internal region of the solenoid with $\rho<R$, and the second one, $D_{2}$, is the external region of the solenoid, with $R \leq \rho<L$, as indicated in figure (2). Now, the vector potential (2.2) is of class $C^{1}$ in both regions, $D_{1}$ and $D_{2}$, and we can apply Stokes' Theorem in each of them. The problem presented in (ii) can be easily circumvented by using a Cartesian coordinate system in the internal region of the solenoid, and dividing the external region into two sectors, one of them with $0 \leq \phi<\pi$ and the other with $\pi \leq \phi<2 \pi$. With this procedure, it can be shown that point (ii) is irrelevant to this problem, and we can apply Stokes' Theorem without taking that into account.

For $D_{1}$, we have the magnetic flux

$$
\Phi_{1}=\iint_{D_{1}} \mathbf{B}_{I} \cdot d \mathbf{S}_{1}=\oint_{\Gamma_{I}} \mathbf{A}_{I} \cdot d \ell=\pi B R^{2}
$$

where $\Gamma_{I}$ is the path around the boundary of the $D_{1}$ region, with positive orientation, as indicated in figure (2). The region $D_{2}$ has two boundaries; in this case, we have

$$
\Phi_{2}=\iint_{D_{2}} \mathbf{B}_{E} \cdot d \mathbf{S}_{2}=\oint_{\Gamma_{L}} \mathbf{A}_{E} \cdot d \ell-\oint_{\Gamma_{R}} \mathbf{A}_{E} \cdot d \ell=0
$$

where we used the property (2.3). 
The total magnetic flux through the whole region $D$ is

$$
\Phi=\Phi_{1}+\Phi_{2}=\pi B R^{2}
$$

With the appropriate choice of the $\gamma$-parameter, as made by Aharonov and Bohm [4], in equation (2.2) we set

$$
\gamma=\frac{B R^{2}}{2}
$$

we have from (2.3),

$$
\oint_{\Gamma_{I}} \mathbf{A}_{I} \cdot d \ell=\oint_{\Gamma_{L}} \mathbf{A}_{E} \cdot d \ell=\oint_{\Gamma_{R}} \mathbf{A}_{E} \cdot d \ell=\pi B R^{2}
$$

With this peculiarity, and equations (2.4), (2.5) and (2.6), we can write

$$
\Phi=\iint_{D} \mathbf{A} \cdot d \mathbf{S}=\oint_{\Gamma_{L}} \mathbf{A}_{E} \cdot d \ell=\oint_{\Gamma_{L}} \mathbf{A} \cdot d \ell=\pi B R^{2}
$$

Equation (2.9) has an interesting interpretation: it is like we could apply Stokes' Theorem to the potential

$$
\mathbf{A}(\rho, z, \phi)=\left\{\begin{array}{l}
\frac{B \rho}{2} \hat{\phi}, \rho<R \\
\frac{B R^{2}}{2 \rho} \hat{\phi}, \rho>R
\end{array},\right.
$$

discarding the fact that it is not a $C^{1}$ field in the region $D$; we simply considered the integral along the exterior path $\Gamma_{L}$.

We would like to emphasize that the value of the $\gamma$-parameter (2.7) is only a choice, it is not an imposition of Stokes' Theorem. Another important point to notice is that, with this choice of $\gamma$, we can define a continuous potential (2.10) at $\rho=R$, (if we consider it defined in this region) but its first derivative is still discontinuous, and consequently, it is not a $C^{1}$-field ${ }^{1}$.

In the context of Quantum Mechanics, the situation is not so simple. As pointed out by Aharonov and Bohm [4], in spite of the magnetic field $\mathbf{B}_{E}$ in the exterior region to be zero, a vector potential of the form (2.2) can yield measurable physical phenomena, even with its rotational being zero. These phenomena, usually known as Aharonov-Bohm effects, have their explanations based on the fact that, by virtue of equation (2.2), when we consider wave functions in the exterior region of the solenoid, these ones become no more single-valued functions, acquiring a phase factor when we take a closed path around the solenoid; so, for an wave function $\psi$ outside the solenoid, we have

$$
\begin{aligned}
\psi(r, \phi+2 \pi, z) & =\exp \left(-i q \oint \mathbf{A}_{E} \cdot d \ell\right) \psi(r, \phi, z) \\
& =\exp (-2 \pi i q \gamma) \psi(r, \phi, z)
\end{aligned}
$$

where $q$ is the charge of the considered wave function ${ }^{2}$.

\footnotetext{
${ }^{1}$ In the spirit of the theory of distributions, a vector potential of the form (2.2) can produce a magnetic delta field on the solenoid $(\rho=R)$. In a classical context, it is not a problem, since the magnetic field is given by (2.1) in the physical regions [7]. A similar fact happens in the Dirac quantization, where, on the Dirac's string, the magnetic field diverges $[2,7]$.

${ }^{2}$ These phenomena are also based on the fact that the domain of the wave function (the exterior region) is not simply-connected [8].
} 
The physical quantities introduced by the property (2.11) are proportional to the phase difference $q \oint \mathbf{A}_{E} \cdot d \ell=2 \pi i q \gamma$ and are periodic functions of the parameter $\gamma$, with period given by $1 / q$. If we have $q \oint \mathbf{A}_{E} \cdot d \ell=2 \pi i q \gamma=2 \pi N$, for $N=0, \pm 1, \pm 2, \ldots$, the wave function (2.11) becomes single valued and we have no physical effect.

In order to keep the vector potential continuous over the whole space, and to keep the familiar expression (2.9) for Stokes' Theorem, as already said, Aharonov and Bohm took the vector potential as being the one expressed in equation (2.10), which turns equation (2.11) into the form

$$
\psi(r, \phi+2 \pi, z)=\exp \left(-i q \pi B R^{2}\right) \psi(r, \phi, z),
$$

where we used equation (2.9).

Ever since, many other problems dealing with an infinitely long solenoid, as discussed above, were studied in the literature, and in all of them, it is considered the vector potential with the form (2.10). As a consequence, the physical result predictions for all of these problems are proportional to the phase difference presented in (2.12).

Different choices for the value for the $\gamma$-parameter in equation (2.2) would give different physical results for effects of the Aharonov-Bohm kind. In spite of this, Stokes' Theorem and Quantum Mechanics do not state that the value of $\gamma$ must be the one expressed in equation (2.7); it was simply a choice.

Nowadays, there are experimental evidences for the existence of Aharonov-Bohm effects, in agreement with the value (2.7). What we wish to explore in this text is the fact that (2.7) is not the only choice that can be done for the $\gamma$-parameter according to the experiments. There is still a certain freedom in the value of $\gamma$. In order to study this point and its consequences, instead of (2.7), let us write:

$$
\gamma=\frac{B R^{2}}{2}+\kappa
$$

where $\kappa$ is any real constant. Inserting (2.13) into equation (2.2), and using (2.11), we have

$$
\psi(r, \phi+2 \pi, z)=\exp \left(-i q \pi B R^{2}\right) \exp (-2 \pi i q \kappa) \psi(r, \phi, z) \text {. }
$$

We do not expect that the choice (2.13) gives physical results different from the ones presented in the literature, and obtained, with the choice (2.7) those for which there are experimental verifications. To assure this, the phase difference of the wave function in (2.14) must be the same phase difference of the equation (2.12). This condition is satisfied by taking

$$
q \kappa=n_{q, \kappa},
$$

where $n_{q, \mathrm{~K}}$ is an integer number that depends on the charge $q$ of the wave function, and on the parameter $\kappa$. In order to get the possible values of $\kappa$, we consider the expression above for the case of the electron charge, $e$. The result is

$$
\kappa=\frac{n_{e, \mathrm{\kappa}}}{e}=\frac{N_{\mathrm{\kappa}}}{e},
$$

where $N_{\kappa}$ is an integer that depends only on $\kappa$. 
Using equations (2.15) and (2.16), we have that any given value of electric charge must satisfy the condition

$$
q=\frac{n_{q, \kappa}}{N_{\kappa}} e .
$$

The only way to avoid a $\kappa$-dependence for the charge $q$ is to take $n_{q, \kappa}=n_{q} n_{\kappa}$ and $N_{\kappa}=N n_{\kappa}$, where $n_{q}$ and $n_{\kappa}$ are integers that depend, respectively, on $q$ and $\kappa$, and $N$ is an integer constant. This finally leads to

$$
q=\frac{n_{q}}{N} e
$$

that is, any value of electric charge $q$ is an integer multiple of a given fraction of the electron charge e.

It is interesting to notice that the result (2.18) is consistent with the existence of anti-particles and with the quarks charges. From equation (2.16), we can see that the possible values of the $\kappa-$ parameter, that was completely free at the classical level, become quantized in the quantum context.

The result (2.18) could be proposed by means of a slightly different point of view. Equation (2.13) is equivalent to a gauge transformation of the form

$$
\mathbf{A} \rightarrow \mathbf{A}+\frac{\kappa}{\rho} \hat{\phi}
$$

for the vector potential (2.10) in the exterior region of the solenoid. This gauge transformation is not in disagreement with Stokes' Theorem and with Quantum Mechanics. In the context of the Classical Electrodynamics, it will produce no effect, but in the context of Quantum Mechanics, it will produce a phase difference of the form (2.14) in a wave function outside the solenoid. As a consequence, we will have, in the problems of the Aharonov-Bohm type, a correction to the physically measurable quantities due only to the gauge transformation (2.19). The only way to avoid physical dependences on the gauge parameter $\kappa$ is to have the condition (2.15). With the previous argument, we are taken to equation (2.18).

A similar gauge transformation to (2.19) occurs in the Dirac's quantization and it is produced in considering that the vector potentials generated by two Dirac's strings, related to the same magnetic monopole, but located in different regions of space, can be related by a gauge transformation of the form (2.19).

In our case, the transformation (2.19) occurs naturally, due only to the fact that it is welldefined in the exterior region of the solenoid because the $z$-axis is excluded from this region (what makes it a region with a non trivial geometry). A similar analysis could be carried out from other situations where we have non-trivial geometries.

As a last comment, we would like to say that the freedom in the $\gamma$-parameter in equation (2.2), or equivalently, the gauge transformation (2.19), was used, some years ago, to argue that the Aharonov-Bohm does not exist [9]. This idea was refused by some authors with the argument that the transformation (2.19) was in disagreement with the Stokes' Theorem [10].

\section{Conclusions and final remarks}

We have shown that Stokes' Theorem allows us to have, in the Aharonov-Bohm experiment, a vector potential outside the solenoid, whose circulation around a closed path is not equal to the flux 
of the magnetic field through a surface whose boundary is the considered path. We have shown that, when we consider this possibility in the context of Quantum Mechanics, by choosing conveniently the vector potential in the exterior of the solenoid, we are taken straight to a quantization condition for the values of the electric charges, without appealing to the existence of magnetic monopoles.

The convenient choice for the vector potential quoted above can be interpreted as a gauge transformation, which has the form of an Aharonov-Bohm potential. The quantization condition attained thereby is consistent with the quark electric charges and with the existence of antiparticles.

We think that these results can be extended to the case of non-Abelian fields, where a quantization condition for the color charges must be obtained too.

\section{Acknowledgements}

The authors would like to thank M. V. Cougo-Pinto, C. Farina, H. Boschi-Filho and F.E. Barone for useful discussions. Professor R. Jackiw is kindly acknowledged for relevant comments, and Professor Nathan Lepora for the help with relevant references. F.A.B. thanks FAPERJ for the invaluable financial help.

\section{References}

[1] P.A.M. Dirac, Quantized Singularities in the Electromagnetic Field, Proc. Roy. Soc. A 133 (1931) 60; P.A.M. Dirac, The Theory of Magnetic Poles, Phys. Rev. 74 (1948) 817.

[2] P. Goddard and D. Olive, Magnetic Monopoles in Gauge Field Theories, Rep. Prog. Phys. 41 (1978) 1361.

[3] Juan A. Mignaco, Electromagnetic Duality, Charges, Monopoles, Topology, ..., Braz. J. of Phys. 31 (2001) 235.

[4] Y. Aharonov and D. Bohm, Significance of Electromagnetic Potentials in the Quantum Theory, Phys. Rev. 115 (1959) 485; Y. Aharonov and D. Bohm, Further Considerations on Electromagnetic Potentials in the Quantum Theory, Phys. Rev. 123 (1961) 1511.

[5] M. Peshkin and A. Tonomura, The Aharonov -Bohm Effect Lectures Notes in Physics 340, Springer-Verlag, Berlin 1989.

[6] J.E. Marsden and A.J. Tromba, Vector Calculus, W.H. Freeman and Company, New York 1996.

[7] F.A. Barone and J.A. Helayël-Neto, work in progress.

[8] R.J. Rivers, Path Integral Methods in Quantum Field Theory, Cambridge University Press, Cambridge 1988.

[9] P. Bocchieri and A. Loinger, Nonexistence of the Aharonov-Bohm Effect, Nuovo Cimento 47 (1978) 475; P. Bocchieri, A. Loinger and G. Siragusa, Nonexistence of the Aharonov-Bohm Effect - II, Nuovo Cimento 51 (1979) 1.

[10] D. Bohm and B.J. Hiley, On the Aharonov-Bohm Effect, Nouvo Cimento 52 (1979) 295; A. Zeilinger, On the Aharonov-Bohm Effect, Lett. Nuovo Cimento 25 (1979) 333; H.J. Rothe, Comments on the Theory of the Aharonov-Bohm Effect, Nuovo Cimento 62 (1981) 54. 